COMMENT. The neuroaxonal dystrophies include the infantile (Seitelberger's disease, late-infantile, and juvenile forms, neuroaxonal leukodystrophy, and Hallervorden-Spatz symdrome) and all are characterized by the spheroids in the terminal endings of axons in the central nervous system. The axonal dystrophy in alpha-Nacetylgalactosaminidase deficiency described in this article differs from that observed in other lysosamal storage diseases. The absence of identifiable lysosomal storage in this newly described disorder suggests a causal relation between the enzyme deficiency and the resultant axonal pathology. A report from the Departments of Pediatrics, Radiology and Neurology, Kyoto, Japan, suggests that magnetic resonance imaging may be useful in the diagnosis and classification of infantile neuroaxonal dystrophy ( $I$ to $M$ et al. Pediatr Neurol 1989; 5:245-8). A six year old boy with typical clinical features of infantile neuroaxonal dystrophy showed increased metal deposition in the globus pallidus and MRI findings of Hallervorden-Spatz syndrame suggesting that these two disease entities overlap. The "eye of the tiger" sign was described in the MRI of two patients with Hallervorden-Spatz syndrome and dystonia. (See Ped Neur Briefs, October 1988, 2:77).

RETT SYNDROME

The clinical peculiarities and differential diagnosis of Rett syndrame are reviewed from the Department of Pediatrics Children's Clinics, East Hospital, Goteborg, Sweden. The four clinical stages of classic Rett syndrame are as follows. 1) Early onset stagnation, 2) rapid developmental regression, 3) pseudostationary period, 4) late motor deterioration. A variety of atypical variants have been described including some in boys. The development during the first few months of life is sometimes abnormal, rarely there is no subsequent deterioration phase, and occasionally seizures occur early as infantile spasms. The term "formes frustes" has been coined for these abortive variants. The differential diagnosis in Rett syndrame stage 2, which includes rapid developmental regression, increased irritability, screaming episodes and loss of acquired skills, is as follows: Infantile neuronal ceroid lipofuscinosis, encephalitis, toxic encephalopathies, epileptic encephalopathies, infantile autism, neurocutaneous syndromes, glutaric aciduria, amino acidopathy, and ataxic cerebral palsy. Biologic markers and effective screening procecures for an early diagnosis are lacking. A possible viral origin with a disorder similar to SSPE is now being suggested as an etiology of this syndrome. (Hagberg BA. Rett syndrome: Clinical peculiarities, diagnostic approach, and possible cause. Pediatr Neurol Mar/Apr 1989; 5:75-83).

COMMENT. As the author concludes, the Rett syndrome concept is broade $\bar{r}$ than previously believed and atypical variants must be recognized. Autopsy findings have been surprisingly limited even in advanced stages of the disease and have included moderate cortical atrophy and general brain shrinking with increasing age. Microscopically, there was mild gliosis without evidence of storage, underpigmentation in certain nigral structures, and axonal changes suggestive of degeneration in ascending and descending tracts. 
Biochemical findings have included a reduction of brain noradrenaline, dopamine and seratonin, but no consistent abnormalities have been found. A genetic basis for Ret $t$ syndrome has been suggested but not satisfactorily confirmed.

\section{MUSCLE DISORDERS}

\section{MITOCHONDRIAL MYOPATHY AND CARDIOMYOPATHY}

Two siblings with infantile lactic acidosis and mitochondrial myopathy are reported from the Department of Pediatrics, Goteborg University; Ostra Hospital; Goteborg, Sweden. The first child, a girl, appeared healthy during the first four months of 1 ife. She was admitted at five months of age with feeding difficulties, vamiting and weight loss and muscular hypotonia. Her serum lactate concentration rose to $20 \mathrm{mmol} / \mathrm{L}$ ( $\mathrm{n}: 0.8-1.8 \mathrm{mmol} / \mathrm{L})$, she developed edema, became comatose and died of circulatory failure eight days after admission. At autopsy, the heart was slightly enlarged and the pleurae and pericardium showed clear yellowish fluid. The second patient, the younger brother of patient one, had congenital lactic acidosis but no other symptoms until six months of age when he developed progressive muscle weakness. Treatment with dichloroacetate lowered the serum lactic acid level but did not affect his clinical condition. Cardiamyopathy was diagnosed at 13 months of age and he died of circulatory failure at 29 months. Both patients had mitochondrial myopathy with changes in skeletal muscle and the myocardium. Biochemical investigations of skeletal muscle mitochondria showed deficiencies in cytochrome $c$ oxidase and NADH ferricyanide recuctase. (Tulinius $\mathrm{MH}$ et al. Mi tochondrial myopathy and cardiamyopathy in siblings. Pediatr Neurol May/June 1989; $\underline{5}: 182-188$ ).

\footnotetext{
COMMENT. Patients with mitochondrial myopathies or cytopathies show marked heterogeneity in clinical manifestations and system involvement. Two major variants of mitochondrial myopathy and cytochrome c oxidase deficiency in infancy have been described. Most cases are rapidly progressive and fatal and are associated with renal dysfunction; occasionally the course is milder and reversible. In the present study, the heterogeneity in the mitochondrial cytochrome c oxidase activity provided clinical symptoms in proportion to the fraction of damaged mitochondria, thus explaining the different clinical course in the siblings.
}

\section{CYTOCHROME C OXIDASE DEFICIENCY AND RESPIRATORY DISTRESS}

A newborn male presenting with severe respiratory insufficiency, generalized muscle weakness, and lactic acidemia is reported from the Department of Pediatrics, Nagasaki University School of Medicine, Japan. Within 27 hours after birth he was markedly hypotonic, spontaneous movements and the Moro reflex were almost absent. A respirator was necessary because of respiratory arrest and he died 75 hours after birth. At autopsy there was variation in muscle fiber size and an increased number of Type $2 \mathrm{C}$ fibers but no ragged-red fibers by Gomori trichrome staining. Biochemical and histochemical studies showed cytochrome c oxidase activity 